\title{
ARTICLE
}

\section{The functions of IL-23 and IL-2 on driving autoimmune effector T-helper 17 cells into the memory pool in dry} eye disease

\author{
Yihe Chen ${ }^{1}$, Chunyi Shao ${ }^{1,2}$, Nai-Wen Fan ${ }^{1}$, Takeshi Nakao ${ }^{1}$, Afsaneh Amouzegar ${ }^{1}$, Sunil K. Chauhan ${ }^{1}$ and Reza Dana (D)
}

Long-lived memory T-helper 17 (Th17) cells actively mediate the chronic inflammation in autoimmune disorders, including dry eye disease (DED). The mechanisms responsible for the maintenance and reactivation of these cells in autoimmunity have been subject of investigation. However, the process through which memory Th17 are generated from their effector precursors remains to be elucidated. Herein, using our murine model of DED, we detect a linear transition from effector-to-memory Th17 cells during the abatement phase of acute inflammation, which is accompanied by persistently high levels of IL-23 and diminished levels of IL-2. In addition, in vitro culture of effector Th17 cells derived from the DED animals with IL-23, but not IL-2, leads to significant generation of memory Th17 cells, along with upregulated expression levels of IL-7R and IL-15R by these cells. Furthermore, supplementation of IL-2 abolishes and blockade of IL-2 enhances IL-23-induced generation of memory Th17 cells in vitro. Finally, in vivo blockade of IL-23 signaling during the contraction phase of primary response inhibits the generation of memory Th17 cells from their effector precursors. Together, our data demonstrate a new dichotomy between IL-23 and IL-2 in driving effector Th17 cells into the memory pool in autoimmune-mediated ocular surface inflammation.

Mucosal Immunology (2021) 14:177-186; https://doi.org/10.1038/s41385-020-0289-3

\section{INTRODUCTION}

Autoimmune disorders are often characterized by their progression from acute inflammation to a persistent chronic stage with low-grade inflammation, interspersed with episodes of flare-ups. ${ }^{1}$ As the acute inflammation abates, the vast majority of effector immune cells undergo apoptosis, while a small fraction survives as memory cells, which mediate acute exacerbations when rechallenged with the same antigen. ${ }^{2,3}$ This immunological memory has been increasingly recognized to play a central role in the chronicity of autoimmune disorders. To date, convincing evidence demonstrates the presence of memory T-helper 17 (Th17) cells in fungal infections ${ }^{4,5}$ as well as autoimmunity and chronic inflammation. ${ }^{6,7}$ Furthermore, recent work by our group has demonstrated a pathogenic population of memory Th17 cells, characterized as $\mathrm{CD} 62 \mathrm{~L}^{-} \mathrm{CD} 44^{\mathrm{hi}} \mathrm{IL}-17^{+} \mathrm{CD} 4^{+}$, in a murine model of dry eye disease (DED), ${ }^{8}$ a highly prevalent and chronic ocular surface inflammatory disorder, ${ }^{9}$ which is principally mediated by the memory Th17 cells identified in our study.

The persistence of pathogenic memory cells and their ability to initiate the recall response are responsible for the chronic and relapsing nature of autoimmune disorders. Recent studies have investigated the signals required for the maintenance and reactivation of memory Th17 cells. Our most recent work has shown that both IL-7 and IL-15 are required for the maintenance of pathogenic memory Th17 cells (which express high levels of receptors for both cytokines), and persistence of the chronic ocular inflammation in DED. ${ }^{10}$ IL-23 has been found essential for achieving a fully functional Th17 recall response primarily through mediating the conversion of memory Th17 cells to effector Th17 and IFN- $\gamma$-producing Th17/1 cells, which lead to exacerbated inflammation. 2,11,12 Despite substantial advances in our understanding of Th17 immunity, there remains a gap in our knowledge regarding the mechanisms through which memory Th17 cells are generated from their effector precursors.

In this report, we use our autoimmune DED model to recapitulate the contraction phase of the primary Th17 immune response, and identify the critical factors that regulate effector Th17 cells to enter the memory pool during this window. We show that levels of both IL-23 and IL-2 are increased in the acute stage of the disease (expansion phase). Following abatement of acute inflammation (contraction phase), IL-23 is maintained at high levels, but IL-2 declines to baseline levels, a phenomenon that is accompanied by generation of memory Th17 cells. Our results demonstrate that in vitro culture of effector T cells isolated at the expansion phase of primary response with IL-23 leads to significant generation of memory Th17 cells via upregulation of both IL-7R and IL-15R. Furthermore, while addition of IL-2 abolishes the IL-23-driven memory cell generation, blockade of IL-2 activity further enhances it. Finally, we observe that in vivo blockade of IL-23 signaling during the contraction phase of primary response inhibits the generation of pathogenic memory Th17 cells from their effector precursors, and prevents the

\footnotetext{
${ }^{1}$ Department of Ophthalmology, Schepens Eye Research Institute, Massachusetts Eye and Ear Infirmary, Harvard Medical School, Boston, MA 02114, USA and ${ }^{2}$ Department of Ophthalmology, Shanghai Ninth People's Hospital, Shanghai Jiao Tong University School of Medicine, Shanghai, China

Correspondence: Reza Dana (reza_dana@meei.harvard.edu)

These authors contributed equally: Yihe Chen, Chunyi Shao
}

Received: 10 September 2019 Revised: 22 March 2020 Accepted: 13 April 2020

Published online: 23 April 2020 
a

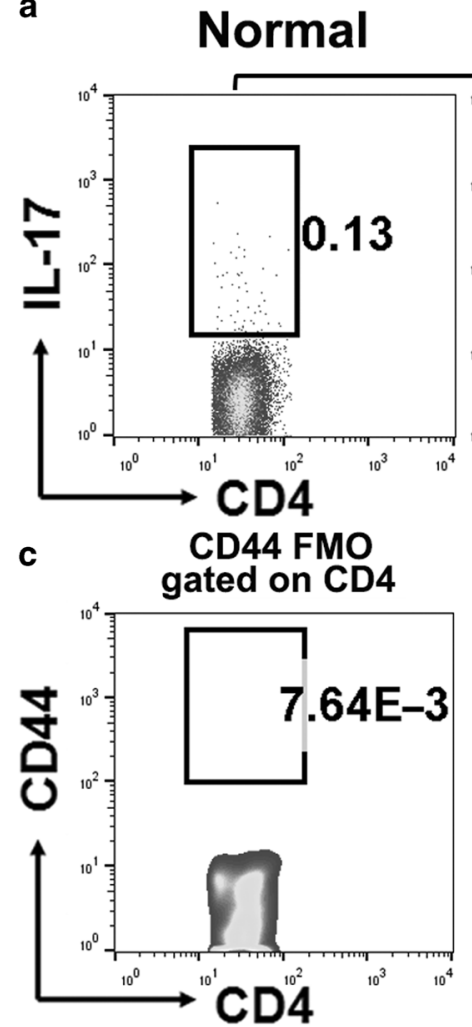

DED - D14 CD4 gated
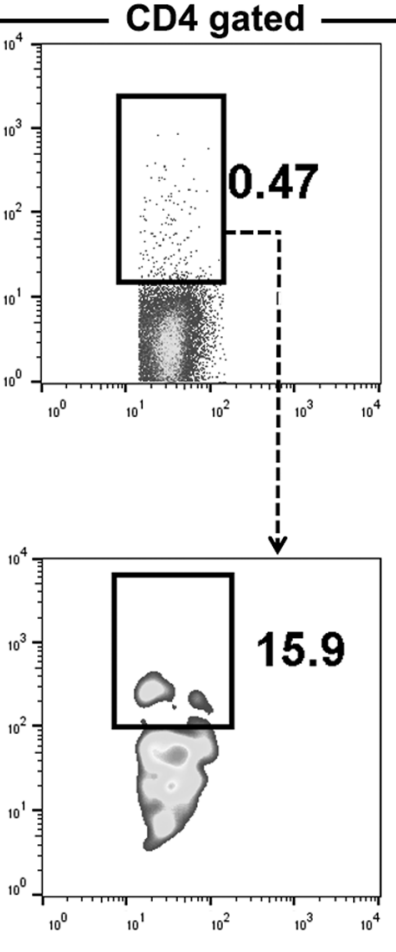

DED- D21

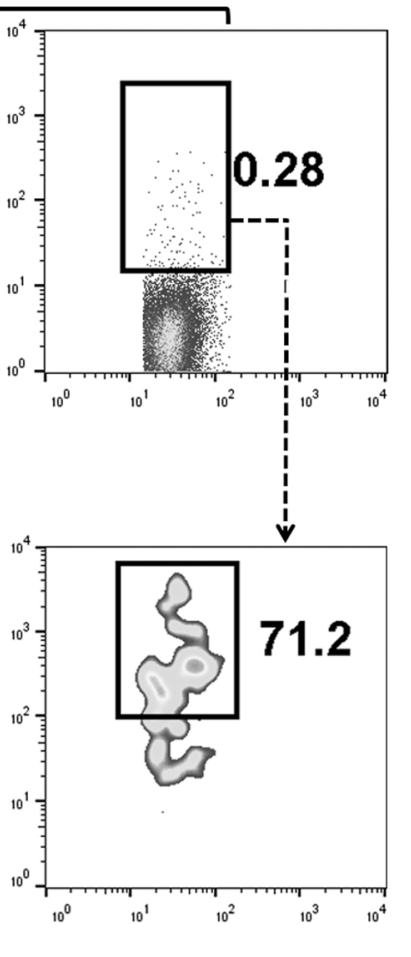

b

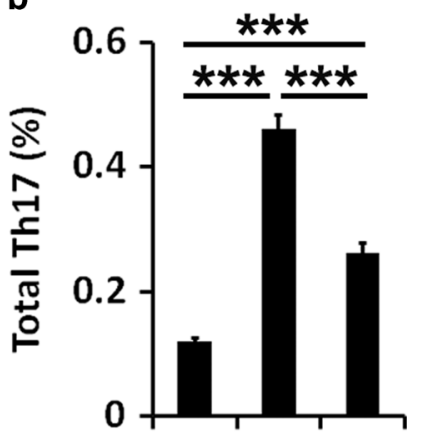

NL D14 D21

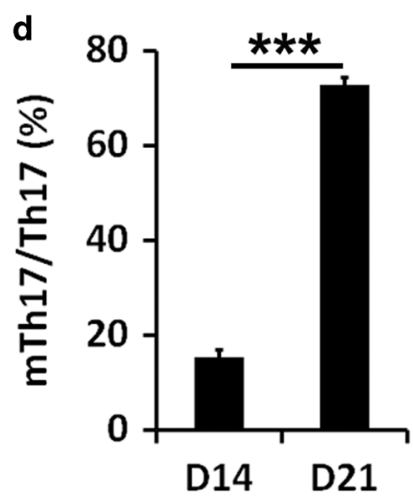

Fig. 1 Generation of memory Th17 during the contraction phase of primary immune response. Mice were challenged with desiccating stress for 14 days to induce dry eye disease (DED), and were subsequently housed in normal vivarium for additional 7 days. Total Th17 $\left(\mathrm{IL}-17^{+} \mathrm{CD} 4^{+}\right.$) cells in draining lymph nodes were examined at D14 (peak of acute inflammation) and D21 (transition to the chronic stage) by flow cytometry with representative plots shown in (a) and summary analysis from 4 mice/group shown in (b). Th17 cells are gated on CD4 ${ }^{+}$ cells. c, d Effector $\left(\mathrm{CD} 44^{\mathrm{lo}}\right.$ ) and memory (CD44 ${ }^{\mathrm{hi}}$ ) Th17 cells among total Th17 cells were further examined at D14 and D21 by flow cytometry. Data shown in the bar graphs represent the mean \pm SEM of a single experiment out of three performed. ${ }^{* *} p<0.001$ by one-way ANOVA followed by Bonferroni's multiple comparisons post hoc test (b) or by Student's $t$ test (d).

progression of disease into the chronic stage. These data highlight a newly described dichotomy between IL-23 and IL-2 in driving effector Th17 cells into the memory pool.

\section{RESULTS}

The contraction phase of primary Th17 response is characterized by generation of memory Th17 cells along with sustained levels of IL-23 and diminished levels of IL-2

The preponderance of evidence suggests that memory CD4 ${ }^{+}$ $T$ cells are generated in a linear effector-to-memory pathway. ${ }^{13-15}$ Various cues from the microenvironment including different cytokines determine the fate of $T$ cells at each phase of the adaptive immune response. We used our murine model of Th17mediated $\mathrm{DED}^{8,10}$ to delineate the characteristics of contraction phase of the primary Th17 response. As shown in Fig. 1, following acute desiccating stress, there was a significant generation of Th17 cells at day 14, of which the predominant cells were CD44 ${ }^{\text {lo }}$ effector Th17 cells. After removal of the desiccating stress and transfer of animals to normal conditions for 7 days, we observed the persistence of Th17 cells (Fig. 1a, b) which, however, consisted of predominant CD44 ${ }^{\text {hi }}$ memory Th17 population (Fig. 1c, d), indicating the conversion of effector Th17 cells dominantly present in expansion phase to memory Th17 cells. Meanwhile, the emergence of these long-lived memory cells was accompanied by the entrance of disease into a chronic stage, which was evident from persisting corneal epitheliopathy. 8,10

We next set to determine the critical factors mediating the conversion of effector Th17 to memory cells. To investigate this, we used a multiplex protein analysis to profile the Th17-pathway cytokines in mice with acute DED. We found that in addition to increased levels of Th17-deriverd pathogenic cytokines, such as IL-17, levels of both IL-23 and IL-2 were increased in the acute disease (Supplementary Fig. 1). To further assess the kinetics of IL-23 and IL-2, we examined them at both the expansion and contraction phases using real-time RT-PCR and ELISA. Our results demonstrated that levels of both IL-23 and IL-2 were significantly increased in the acute disease, when effector Th17 response reached its peak (day 14) (Fig. 2a, b). However, during the contraction phase when prominent memory Th17 developed (day 21) and thereafter, IL-23 was persistently maintained at high levels, while IL-2 returned to its baseline levels (Fig. 2a, b). We observed similar changes in IL-23 and IL-2 levels at the ocular surface (Fig. 2c, d), where infiltration of pathogenic effector or memory Th17 cells occurs at the acute and chronic stages of DED, respectively. 8,10

IL-23 promotes, but IL-2 inhibits ex vivo generation of memory Th17 cells

As a critical cytokine in Th17-mediated immunity, IL-23 is required for Th17 priming and development of a robust recall response in experimental autoimmune encephalomyelitis. ${ }^{11}$ However, the precise role of IL-23 in the conversion of effector Th17 to memory Th17 cells has remained unknown. Knowledge about the function of IL-2 in memory T cell generation is also limited, with one report showing that administration of exogenous IL-2 early after immunization promoted, but an additional dose at a later time point inhibited, development of memory $\mathrm{CD}^{+}{ }^{+}$cells. ${ }^{16}$ Taken 
a

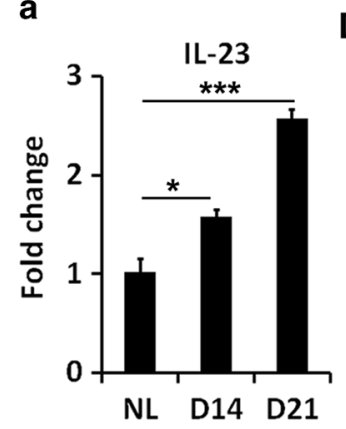

DLN

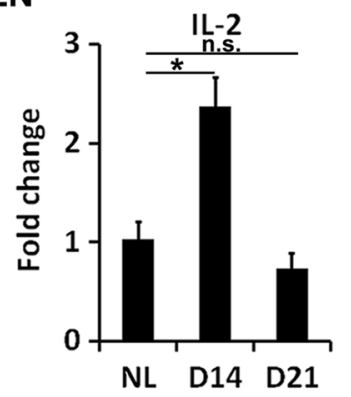

b

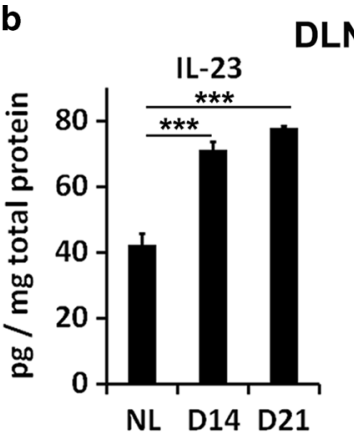

C

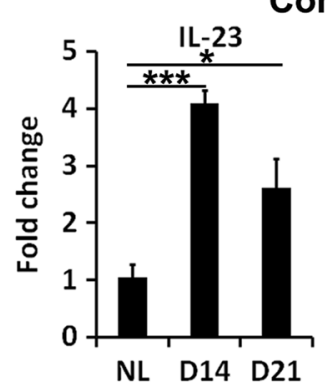

Conjunctivae

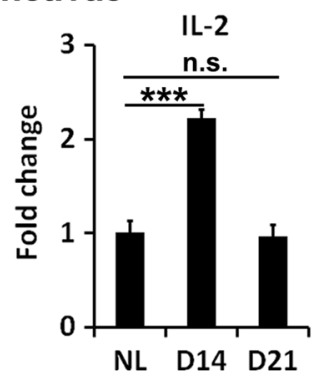

d

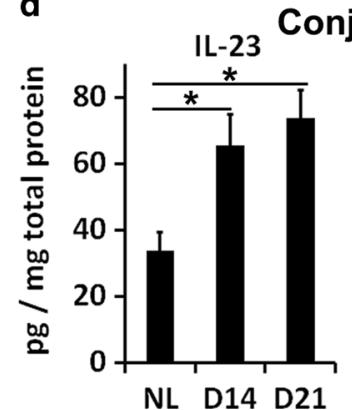

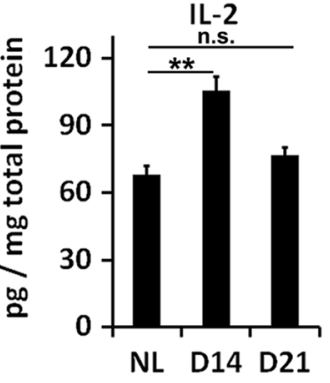

Fig. 2 Memory Th17 cell generation occurs in the IL-23-rich and IL-2-diminished environment. Expression levels of IL-23 and IL-2 in the draining lymph nodes (DLN) of DED mice were quantified using RT-PCR (a) and ELISA (b) at D14 and D21. The mRNA levels were normalized to the housekeeping gene Gapdh and the fold changes were calculated relative to the normal mice $(n=4)$. The protein levels were normalized to the equal amount of the total protein extracted from the tissue $(n=4)$. Expression levels of IL-23 and IL-2 in the conjunctivae (target tissue) of DED mice were quantified using RT-PCR (c) and ELISA (d) at D14 and D21 ( $n=4$ for each analysis at each time point). Data represent the mean \pm SEM of a single experiment that was reproduced in a similar, independent experiment. ns not significant by one-way ANOVA followed by Bonferroni's multiple comparisons post hoc test. ${ }^{*} p<0.05 ;{ }^{* *} p<0.01 ;{ }^{* *} p<0.001$.

together with our findings showing increased IL-23 and reduced IL-2 in the contraction phase, we hypothesized that the generation of memory Th17 from effector precursors is positively regulated by IL-23 and inhibited by IL-2.

To test this hypothesis, we first confirmed that $\mathrm{CD} 44^{\text {lo }} \mathrm{CD} 62 \mathrm{~L}^{-} \mathrm{IL}-$ $17^{+} \mathrm{CD}^{+}$effector Th17 cells from mice with acute DED express high levels of IL-23 and IL-2 receptors (IL-23R and IL-2R) (Fig. 3a). Next, we isolated $\mathrm{CD} 44^{\mathrm{lo}} \mathrm{CD} 62 \mathrm{~L}^{-} \mathrm{CD} 4^{+}$effector T cells from the draining lymph nodes of mice with acute DED, and cultured them in the presence of various cytokine and blocking antibody combinations. After $48 \mathrm{~h}$, cells were collected to evaluate the proportion of $\mathrm{CD} 44^{\text {hi }}$ memory Th17 cells generated in each culturing condition. Our results showed that addition of IL-23 to the culture led to significant recovery of total Th17 cells (Fig. 3b, c) and generation of a prominent memory Th17 population (Fig. 3d, e), while addition of IL-2 did not affect the total recovered Th17 cells (Fig. 3b, c) or frequencies of memory Th17 cells (Fig. 3d, e). Furthermore, exogenous IL-2 was able to abolish the effect of IL23 in inducing memory Th17 generation. In contrast, blockade of IL-2 signaling by adding CD25 (IL-2R a subunit, IL-2Ra) blocking antibody enhanced the generation of memory Th17 cells, possibly via interrupting the autocrine effect of IL-2 secreted from the effector T cells themselves in the culture. Finally, the combination of IL-23 and CD25 blocking antibody resulted in the generation of the highest number of memory Th17 cells (Fig. 3d, e) compared with other groups, indicating the opposing roles of IL-23 and IL-2 in driving and inhibiting the generation of memory Th17 cells from effector precursors.

IL-23 drives Th17 cells to enter the memory pool via up-regulating the expression of IL-7 and IL-15 receptors and preventing cell apoptosis

We next investigated the mechanisms through which IL-23 promotes the generation of memory Th17 cells. Given the critical roles of IL-7 and IL-15 signaling for the maintenance of memory Th17 cells, ${ }^{10}$ we hypothesized that IL-23 drives Th17 cells to enter the memory pool by up-regulating the expression of IL-7R and IL-15R. To test this hypothesis, we examined DED-effector Th17 cultures, and found that IL-23 alone or in combination with CD25 blocking antibody significantly enhanced the expression of IL-7R and IL-15R by $2-3$ folds, and CD25 blocking antibody alone modestly enhanced the expression of these receptors by $\sim 1.5$ folds. While IL-2 alone did not affect the expression of either receptor, it was able to abolish IL-23-induced upregulation of both receptors (Fig. 4a, b).

We next evaluated differential effects of IL-23 and IL-2 on survival of cultured Th17 cells using Annexin V apoptosis detection kit. We found that while IL-2 led to a significant increase in the frequencies of Annexin $\mathrm{V}^{+}$apoptotic cells, IL-23 alone or in combination with CD25 blocking antibody protected Th17 cells against apoptosis (Fig. 4c).

Blockade of IL-23 signaling during the contraction phase of primary Th17 response leads to reduced memory Th17 generation Having established the important function of IL-23 in driving effector Th17 into memory pool ex vivo, we next assessed whether IL-23 plays the same role in memory Th17 generation in vivo. To this end, we induced a natural, robust effector Th17 response in acute DED (by day 14), and then blocked IL-23 activity using an anti-IL-23R antibody during the contraction phase (days14-21, a period with heightened IL-23 and diminished IL-2 levels in the tissue microenvironment) (Fig. 5a). The anti-IL-23Rtreated group showed a more rapid decline in disease severity throughout our observed duration of 7 weeks post challenge (until day 63), and the disease severity remained stable at a significantly lower level from days 42-63 (Fig. 5b). In addition, we examined the frequencies of memory Th17 cells after the treatment, and we found a significantly reduced memory Th17 
a

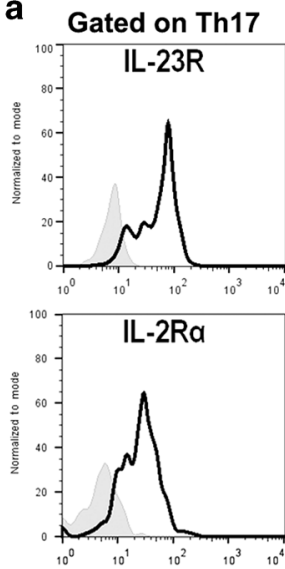

b
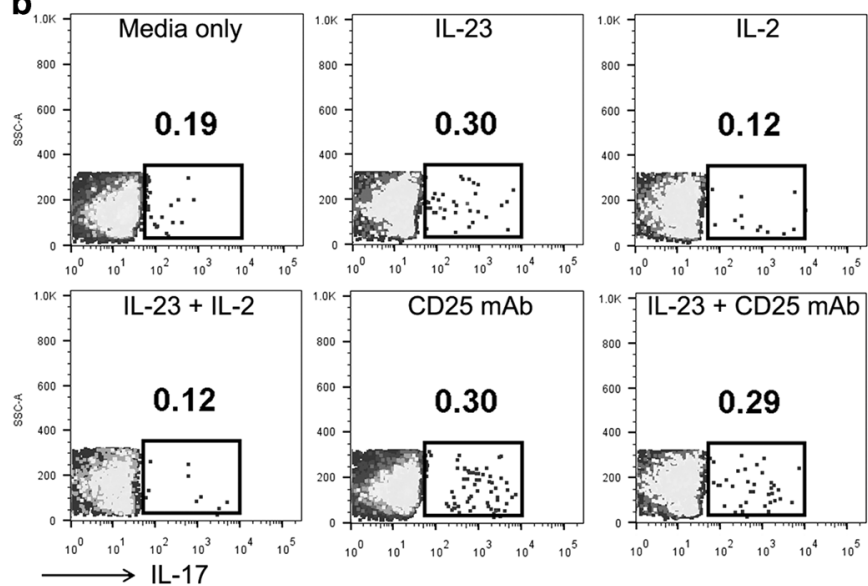

d

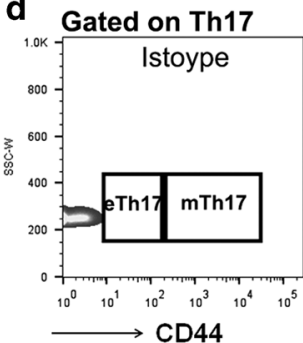

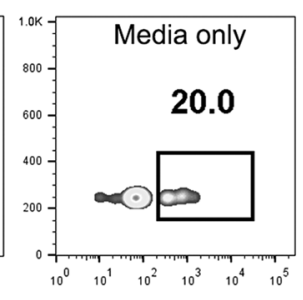

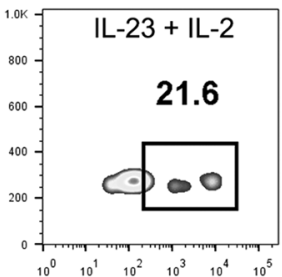

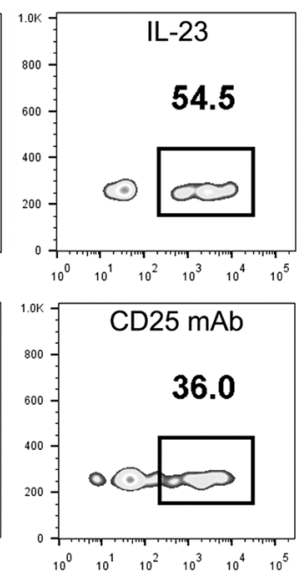

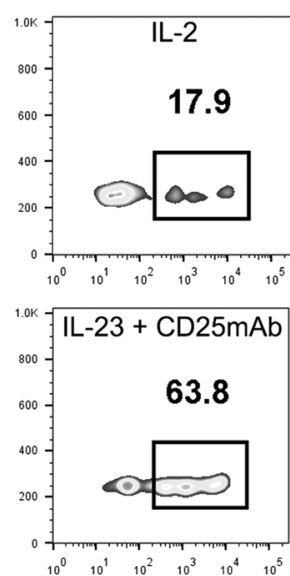

C

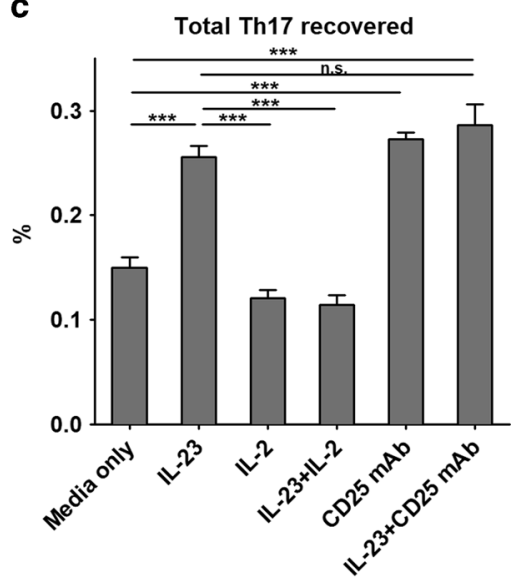

e

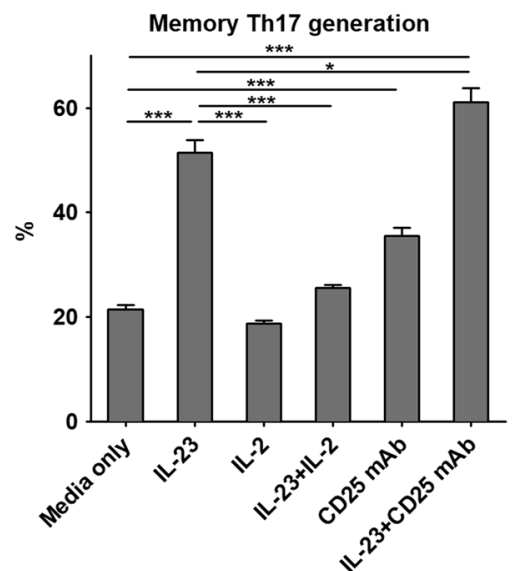

Fig. 3 IL-23 promotes, but IL-2 inhibits generation of memory Th17 from its effector precursor in vitro. a Representative flow cytometry histograms of IL-23R and IL-2R $(\mathrm{CD} 25)$ gated on $\mathrm{CD} 44^{\mathrm{lo}} \mathrm{CD} 62 \mathrm{~L}^{-} \mathrm{IL}-17^{+} \mathrm{CD} 4^{+}$effector Th17 cells from acute DED (D14) mice are shown. Gray histogram is the isotype control. b, c The CD44 ${ }^{\mathrm{lo}} \mathrm{CD} 62 \mathrm{~L}^{-} \mathrm{CD} 4^{+}$effector T cells were sorted from the DLN of acute DED mice (D14) and cultured in the presence of different cytokines and blocking antibody, as indicated in each group for $48 \mathrm{~h}$. The cultured cells were then subject to flow cytometry analysis of total Th17 cells recovered. Representative plots (b) and summary of frequencies (c) are shown. d, e The memory (CD44 ${ }^{\text {hi }}$ ) versus effector $\left(C D 44^{\circ \circ}\right)$ proportions among total Th17 cells were further examined. eTh17 effector Th17, CD25 mAb monoclonal $C D 25$ blocking antibody. Data shown represent mean \pm SEM of three independent experiments with $n=3-4$ for each group in each experiment. ${ }^{*} p<0.05 ;{ }^{* * *} p<0.001$ by one-way ANOVA followed by Bonferroni's multiple comparisons post hoc test.

population at both day 21 and day 63 (Fig. 5c). Next, we evaluated the long-term effect of antibody treatment on diminishing DED pathogenesis by rechallenging DED mice that were treated during the contraction phase with desiccating stress at day 63, but without administering the antibody during the period (Fig. $5 \mathrm{~d}$ ). As compared with the control group, which showed faster disease exacerbation and a more severe disease with the 7-day rechallenge, the group treated with anti-IL-23R antibody during the contraction phase showed no or minimal disease exacerbation upon rechallenge (Fig. $5 \mathrm{~d}$ ). At the end of the clinical follow-up (day 70), the memory-to-effector conversion was examined by analyzing the effector Th17 response. Compared with the expanded Th17 cell population after rechallenge in the control group, the frequencies of Th17 cells in the draining lymph nodes, as well as the levels of IL-17 in the conjunctivae, were significantly lower in the antibody-treated group (Fig. 5e, f). Previous studies have shown that blockade of IL-23 activity during the reactivation of established pathogenic memory Th17 cells impairs the generation of effector cells and diminishes disease relapse. ${ }^{11}$ Similarly, blockade of IL-23 in mice with established chronic arthritis was shown to reduce disease relapse upon re-immunization with the antigen. ${ }^{2}$ Herein, we observed that blockade of IL-23 signaling during the contraction phase (effector-to-memory transition stage, and before establishment of memory) effectively impairs memory Th17 formation and prevents subsequent memory response and disease recurrence, which altogether demonstrates the critical role of IL-23 in both memory Th17 generation and reactivation.

Blockade of IL-23 signaling inhibits in vivo effector-to-memory conversion

A previous study showed that human $\mathrm{IL}-23 \mathrm{R}^{-} \mathrm{CD} 45 \mathrm{RO}^{+} \mathrm{CD} 4^{+}$nonTh17 memory cells can be induced to produce IL-17 through substance P-induced expression of IL-1 $\beta$, IL-23, and TNF-like $1 \mathrm{~A}$ by monocytes, ${ }^{17}$ which raises the possibility that our observations on the effect of in vivo blockade of IL-23 signaling on generation of memory Th17 cells might not be specifically through affecting the effector precursors. To address this question, we used an adoptive transfer approach. Acute DED was induced in wild-type CD45.2 $2^{+}$

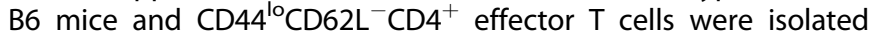
from their draining lymph nodes. Acute DED was also induced in congenic wild-type CD45.1 ${ }^{+}$B6 mice, and at the beginning of the contraction phase (day 14), mice received an intravenous injection of CD45.2 $2^{+}$DED-effector T cells. Immediately after T cell transfer, the recipients received either an anti-IL-23R antibody or isotype lgG. After 7 days, we were able to detect the congenically labeled Th17 cells at the target tissue (conjunctivae) and the draining 
a

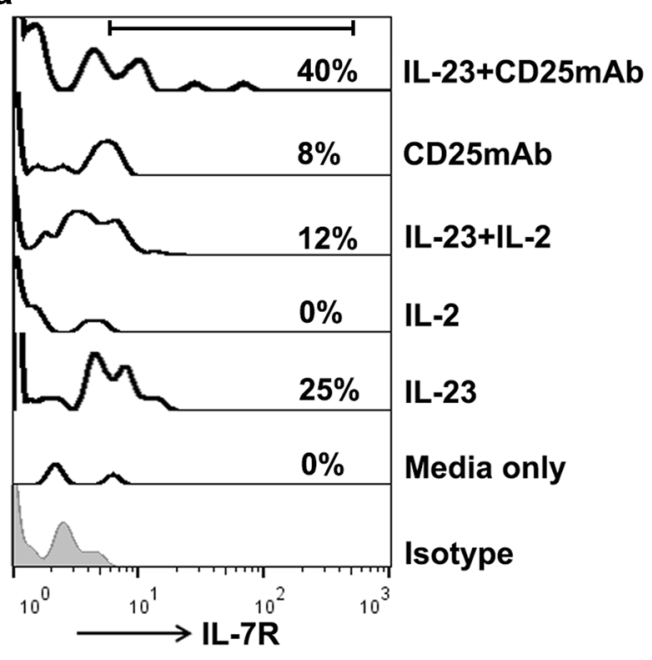

b
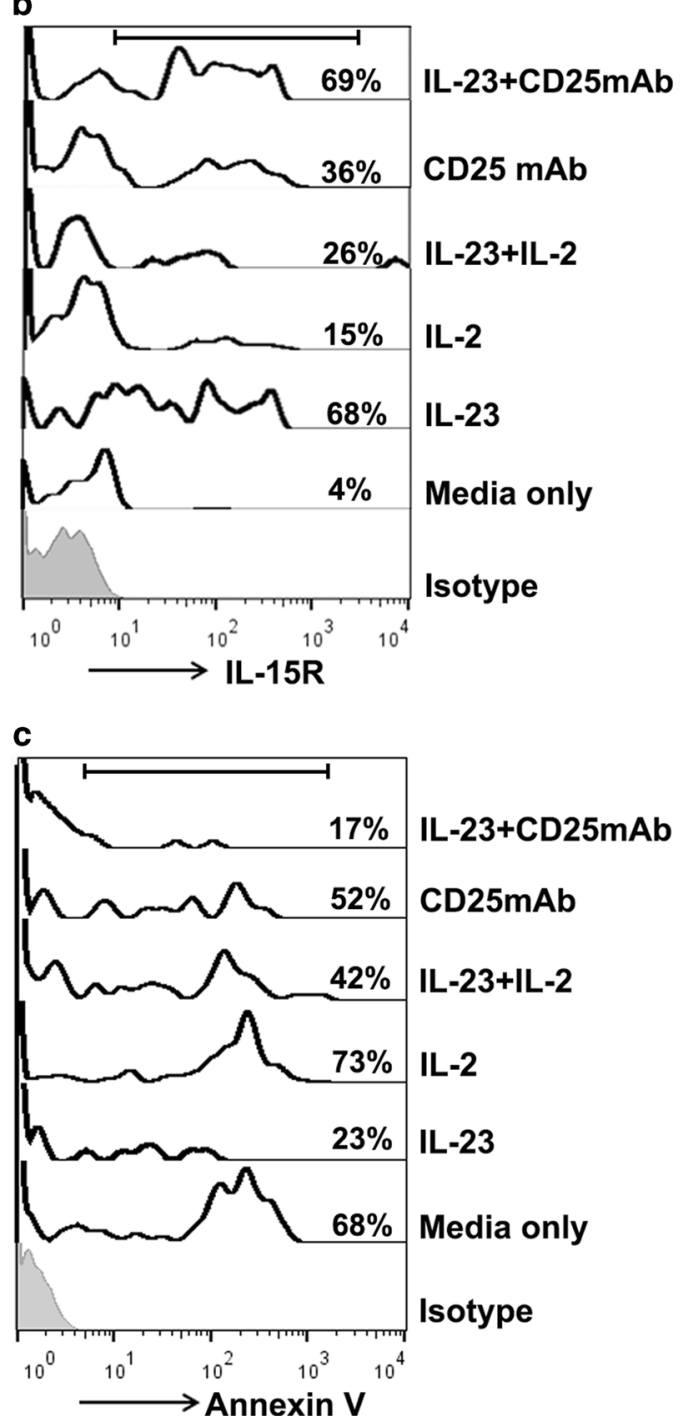
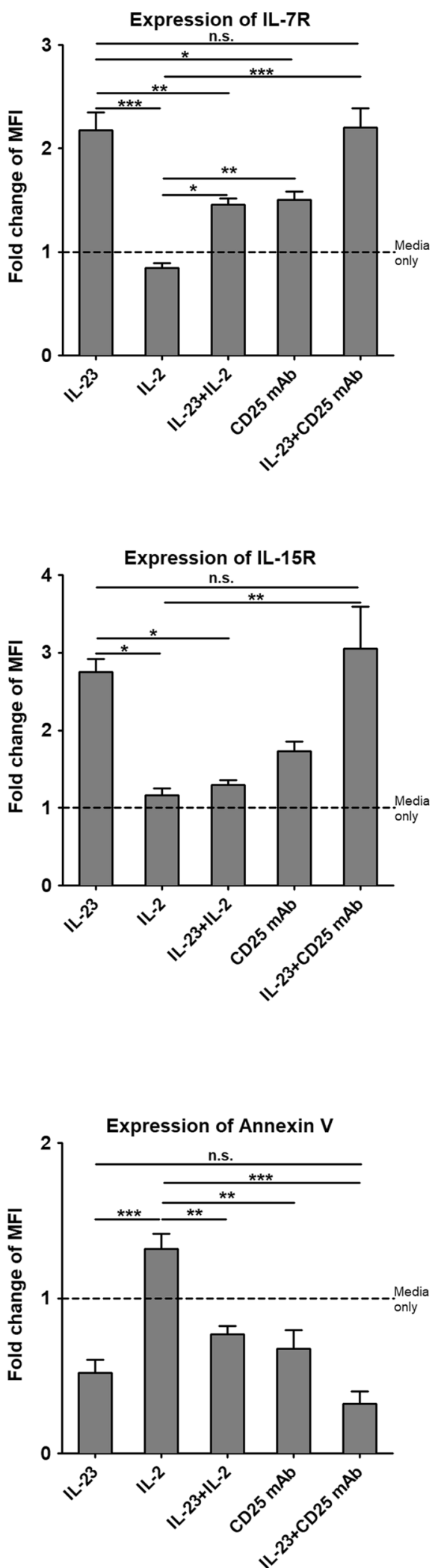

Fig. 4 IL-23 up-regulates expression levels of IL-7R and IL-15R and prevents apoptosis of Th17 cells. The cultured Th17 cells were analyzed for their expression levels of IL-7R (a), IL-15R (b), and Annexin V (c) using flow cytometry. Representative histograms with indicated cell frequencies and quantifications of mean fluorescence intensity (MFI) for expression of each molecule are shown. The fold changes in MFI were calculated relative to the media only group. Data shown represent the mean \pm SEM of three independent experiments with $n=3-4$ for each group in each experiment. ns not significant by one-way ANOVA followed by Bonferroni's multiple comparisons post hoc test. ${ }^{*} p<0.05 ;{ }^{* *} p<$ $0.01 ;{ }^{* * *} p<0.001$. 
a

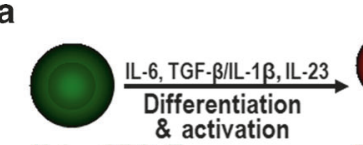
Naive CD4+ T ferentiation
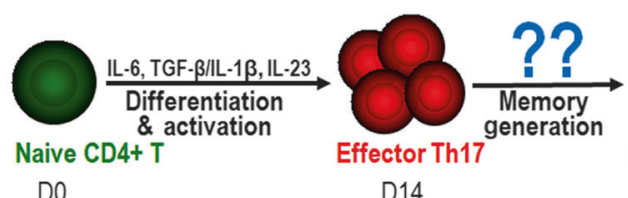

Effector Th17

eneration

D0 D14

B6 mice

D14

Desiccating stress

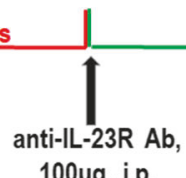

Normal environment
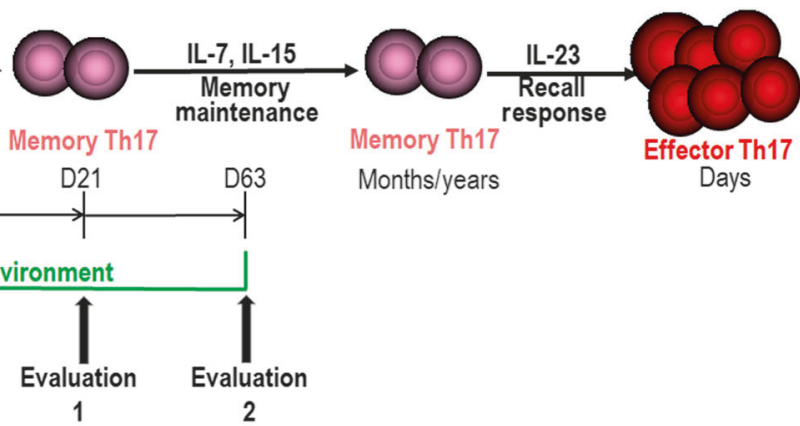

b

2

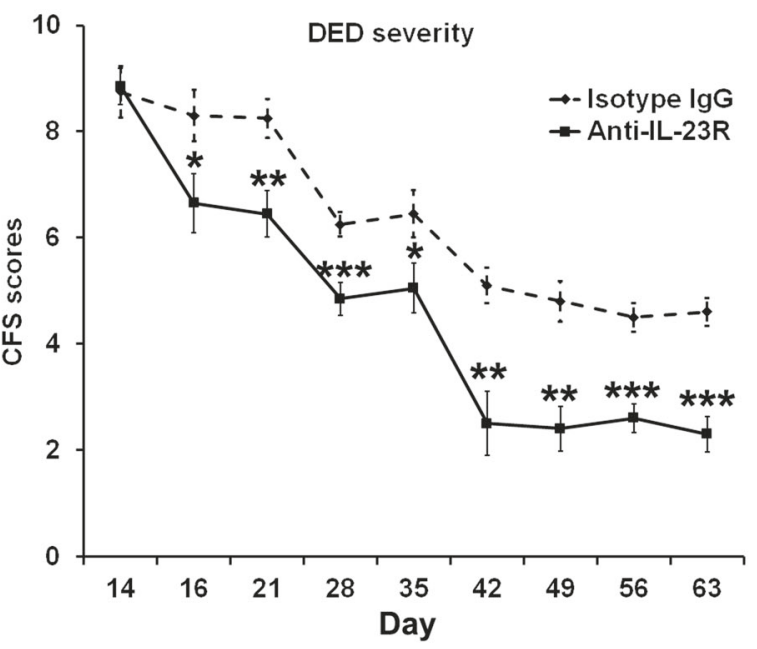

C Gated on CD4

d
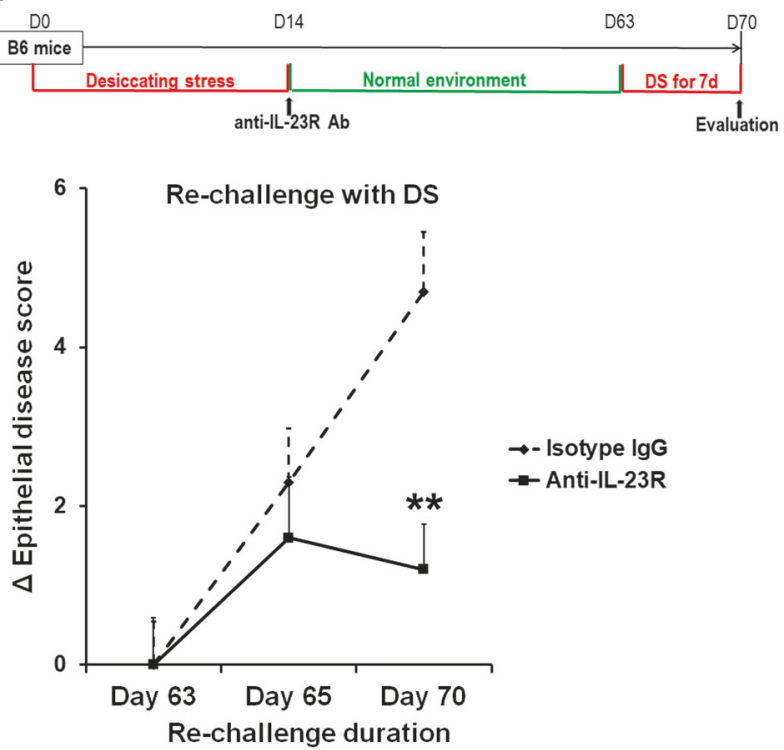

lymph nodes in the control group (Fig. 6a, b, d, e). A modest number of $\mathrm{CD} 45.2^{+}$cells were also detected in the spleen, but none were IL-17-secreting Th17 cells (Supplementary Fig. 2). Anti-IL-23R treatment significantly reduced the total frequency of IL-17-producing congenic cells as well as the proportion of generated congenic IL-7R ${ }^{+}$CD $44^{\text {hi }}$ memory Th17 cells compared with the control group, indicating that in average around $70 \%$ of congenic Th17 cells were memory cells in the draining lymph

Gated on CD4
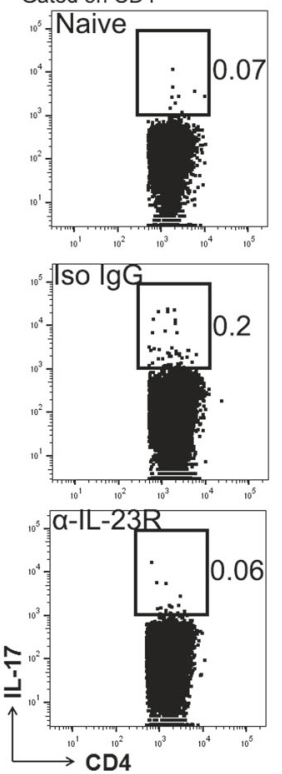

Memory Th17 in DLN

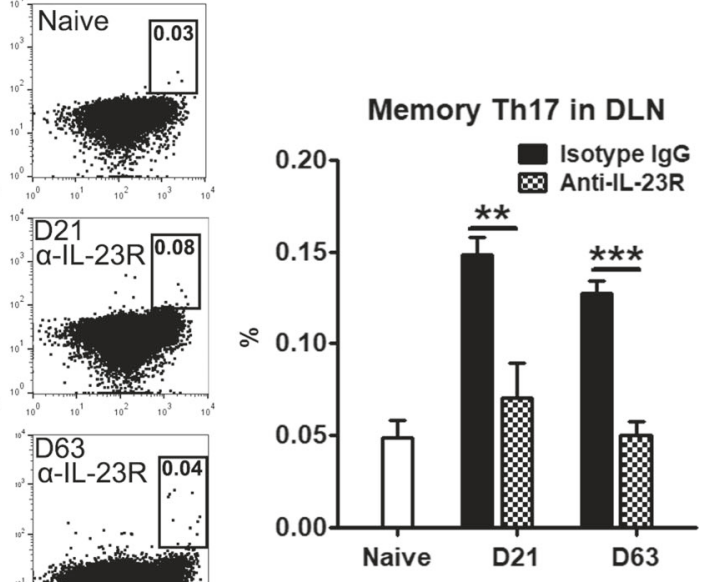

Effector Th17 in DLN
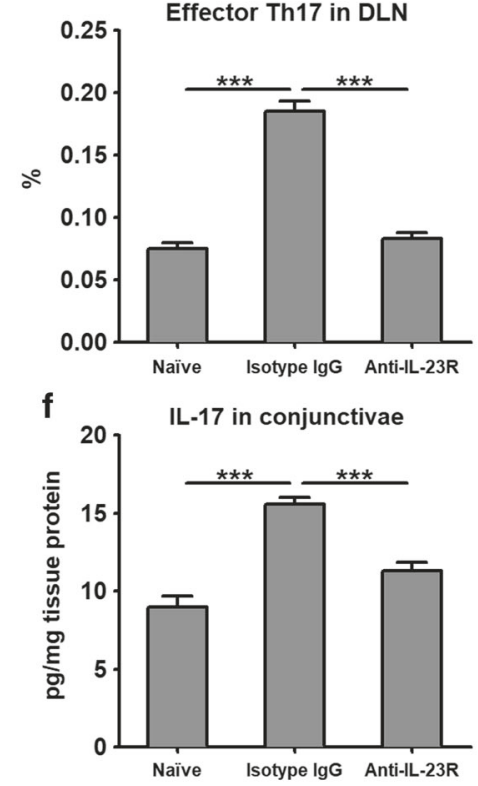
Fig. 5 In vivo blockade of IL-23 during the resolution of acute inflammation leads to reduced memory Th17 pool and recall response. a Schematic study design of in vivo blockade experiment. Mice were challenged with desiccating stress for 14 days to induce acute DED, and were subsequently housed in normal vivarium for the remaining days. At D14, mice were treated with a single dose of $100 \mu \mathrm{g}$ of anti-IL-23R antibody intraperitoneally once they were removed from the desiccating stress. At D63, mice were either sacrificed for memory Th17 analysis or rechallenged with desiccating stress for evaluation of the recall response. $\mathbf{b}$ Clinical disease was evaluated during the contraction phase of primary response (D14-D21) throughout to the chronic phase until D63. Corneal fluorescein staining (CFS) was used to assess disease severity. The mean score \pm SEM pooled from two independent experiments is shown ( $n=20$ eyes). ${ }^{*} p<0.05 ;{ }^{* *} p<0.01$; ${ }^{* * *} p<0.001$ by Student's $t$ test. c Memory Th17 cell frequencies in the draining lymph nodes were analyzed at D21 and D63 by flow cytometry. CD44 versus IL-17 expression by $\mathrm{CD}^{+}$cells is presented on representative plots, and the percentages of memory Th17 cells are summarized as mean \pm SEM of a single experiment out of two performed $(n=5) .{ }^{* *} p<0.01 ;{ }^{* * *} p<0.001$ by one-way ANOVA followed by Bonferroni's multiple comparisons post hoc test. d-f Recall response was evaluated at D63. Previously anti-IL-23R or isotype antibody-treated mice were rechallenged with desiccating stress (DS) at D63 for additional 7 days. Clinical disease was assessed using CFS scoring during D63-D70 with the disease score changes calculated as epithelial disease score = disease score after rechallenge-disease score before rechallenge (d). At D70, Th17 frequencies in the draining lymph nodes were analyzed by flow cytometry (e), and the levels of IL-17 in the conjunctiva were measured by ELISA on the pooled tissue homogenates and normalized to the equal amount of the total proteins (f). Data represent the mean \pm SEM with five animals in each group. ${ }^{* *} p<0.01 ;{ }^{* * *} p<0.001$ by Student's $t$ test (d) or by one-way ANOVA followed by Bonferroni's multiple comparisons post hoc test (e, $\mathbf{f}$ ).

\section{DISCUSSION}

Th17 immunity is the major driver of autoimmune and chronic inflammatory disease, and the formation of Th17 memory is critical for maintaining the chronicity of the disease. ${ }^{6-8,10,11}$ In this study, we demonstrate that an IL-23-enriched but IL-2-diminished microenvironment present during the contraction phase of the autoimmune response will drive previously expanded effector Th17 cells into the memory pool, and that excessive IL-2 signaling or blockade of IL-23 signaling at this phase will inhibit the effectorto-memory conversion of Th17 cells, and prevent the development of subsequent chronic and relapsing disease.

In our Th17-driven autoimmune DED model, ${ }^{8,10}$ we observe simultaneous IL-23 and IL-2 upregulation in the expansion phase, and paradoxically high levels of IL-23 and reduced levels of IL-2 in the contraction phase. These findings reinforce the importance of temporal alterations in cytokine levels in Th17 immune pathway. IL-23 is well-known for its role in promoting Th17-mediated autoimmunity through activating initially differentiated Th17 cells to acquire pathogenicity, and further stabilizing Th17 effectors. $^{2,18-20}$ IL-2 serves as an important autocrine growth factor for effector T cells, and promotes Th1 and Th2 differentiation. $^{21-23}$ However, this is not the case for the Th17 subset, in which IL-2 inhibits the STAT5 signaling-mediated differentiation of these cells from naïve $T$ cells. ${ }^{23,24}$ Interestingly, already differentiated Th17 effectors respond differently and expand in response to IL-2. ${ }^{25}$ Therefore, it is plausible to hypothesize that increased levels of IL-2 observed in acute DED in our study, along with high levels of IL-23, are essential for promoting further proliferation of differentiated Th17 during the expansion phase. However, during the contraction phase, IL-23 is maintained at high levels but IL-2 is reduced, suggesting divergent roles for IL-23 and IL-2 in driving the generation of memory Th17 cells from effector precursors. We indeed further demonstrate that IL-23 promotes, but IL-2 inhibits the conversion of effector Th17 to memory Th17 cells.

It has been reported that there is increased Th17 cell apoptosis after treatment with anti-IL-23 antibody ${ }^{26}$ and high susceptibility of IL-23R-deficient IL-17 ${ }^{+}$T cells to apoptosis, ${ }^{20}$ suggesting an association between IL-23 and Th17 cell apoptosis. Our study provides new evidence indicating the direct role of IL-23 in driving Th17 memory formation via promoting the expression of IL-7R and IL-15R by Th17 cells and preventing their apoptosis. It also suggests that IL-23, which functions primarily as a driver of Th17 immunity, promotes the Th17 response through differential roles in various stages of the immune response, with predominantly proliferative role in the expansion phase versus antiapoptotic role in the contraction phase.

The precise pathways through which IL-23 regulates IL-7R and IL-15R expression in Th17 cells are yet to be investigated. IL-2 has been shown to induce upregulation of IL-2Ra, ${ }^{27}$ and expression of
IL-2Ra after T cell priming is accompanied with the loss of IL-7R by T cells, ${ }^{28}$ suggesting that our observed effect of IL-2 in abolishing the antiapoptotic function of IL-23 in cell cultures could due to IL2-induced upregulation of IL-2Ra on Th17 cells. In contrast to our findings, a previous study using IL-2 ${ }^{-/}$T cells found that high IL-2 signals during a late time window after initial priming were required for generation of memory $T$ cells by preventing effector $T$ cell apoptosis. ${ }^{29}$ This discrepancy could be due to the inherent deficiency in IL-2 autocrine effect on these IL-2-1- T cells during their in vivo development. In addition, this study used a viral infection model, and the principal $\mathrm{CD}^{+}{ }^{+} \mathrm{T}$ cells recovered were IFN- $\gamma$-producing Th1 cells, which could have cytokine-mediated signal requirements different from that of Th17 cells to develop to memory cells. IL-2 has indeed been shown to play opposite roles in the differentiation of Th1 and Th17 cells. ${ }^{21,23,24}$ IL-2 signaling during Th17 priming is essential for the subsequent generation of memory $\mathrm{T}$ cells, due to its role in early cell programming and generation of a larger effector pool. However, our data demonstrate that continuous exposure of effector Th17 to IL-2 prevents their further conversion into memory cells, mediated by IL-23, at least partially through its induction of cell apoptosis (Fig. 4c). Consistent with our findings, studies have shown that early heightened IL-2 signaling during priming followed by a diminishment in IL-2 levels is essential for effector $\mathrm{CD} 8^{+} \mathrm{T}$ cells to give rise to memory $\mathrm{CD} 8^{+}$T cells. $^{30}$ However, the precise cross talk between IL-2 and IL-23 signaling during this process remains to be elucidated. Overall, our findings reveal previously undescribed roles for IL-23 and IL-2 in the generation of memory Th17 cells, and provide a new perspective on mechanisms that drive effector Th17 cells into the memory pool in DED and other chronic Th17mediated disorders.

\section{METHODS}

Animals

Six- to 8-week old female C57BL/6 mice (Charles River Laboratories) and congenic strain B6 CD45.1 (B6.SJL-Ptprc ${ }^{\mathrm{a}}$ Pepc ${ }^{\mathrm{b}} /$ BoyJ) mice (The Jackson Laboratory) were used in this study. All animal experiments were approved by the Institute Animal Care and Use Committee, and adhered to the Association for Research in Vision and Ophthalmology Statement for the Use of Animals in Ophthalmic and Vision Research.

\section{Experimental model of autoimmune DED}

DED was induced in mice, as previously described with few modifications. ${ }^{8,10}$ In brief, following the initial 14 days of exposure to desiccating stress in a controlled-environment chamber (relative humidity: $<20 \%$, dry airflow: $15 \mathrm{~L} / \mathrm{min}$, temperature: $21-23^{\circ} \mathrm{C}$ ), mice were housed in the standard non-desiccated vivarium (relative humidity: $40-60 \%$, no airflow, temperature: 
a

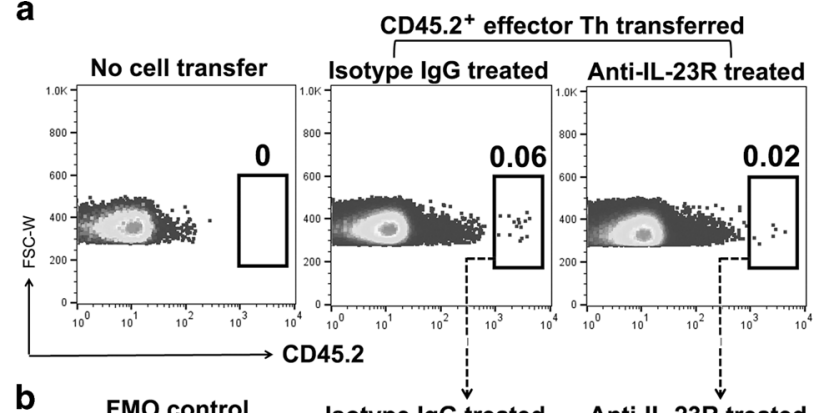

b FMO control Isotype IgG treated Anti-IL-23R treated

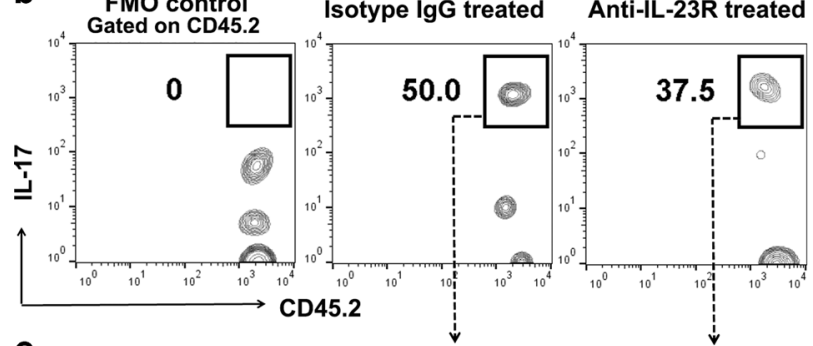

C FMO control

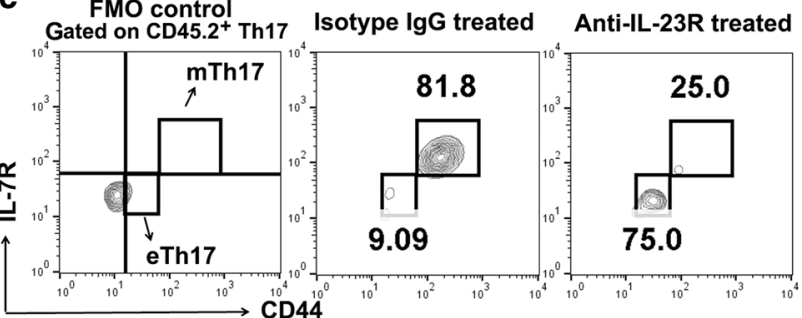

d

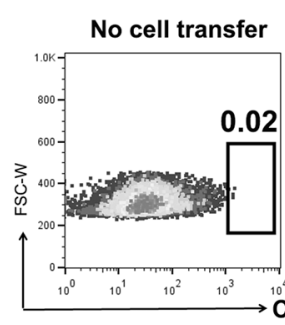

e FMO control

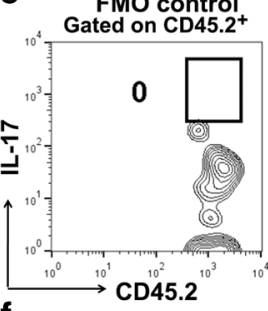

f Gated on CD45.2+ Th17

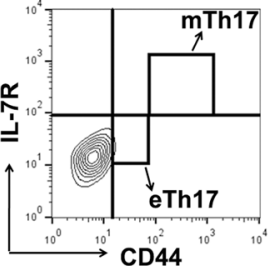

CD45.2 ${ }^{+}$effector Th transferred

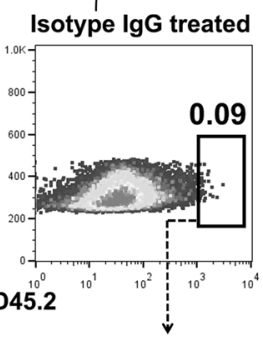

Isotype IgG treated

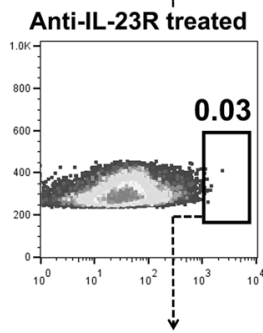

Anti-IL-23R treated
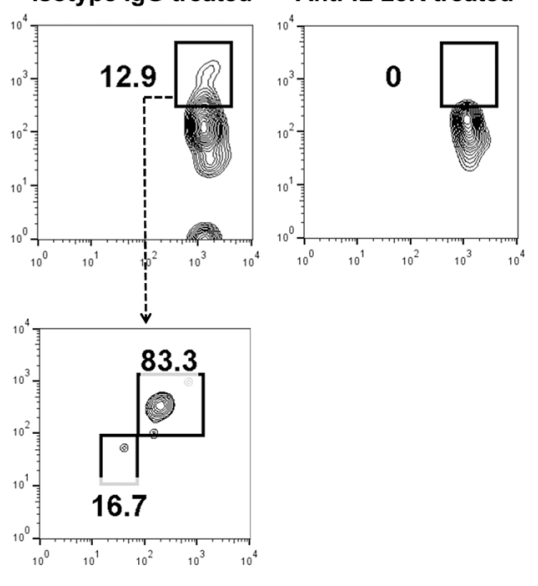
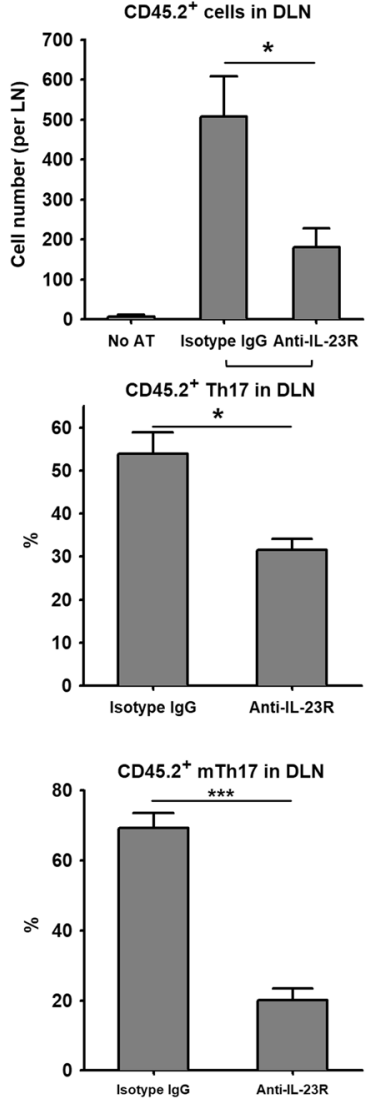

Cell number in conjunctivae
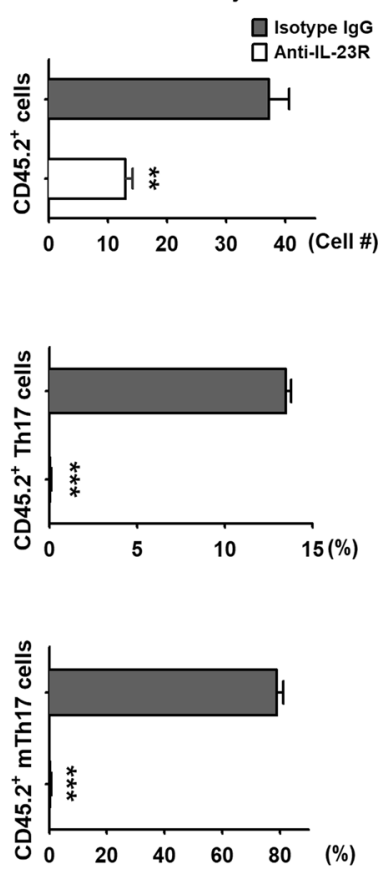

$21-23^{\circ} \mathrm{C}$ ) for an additional period of up to 7 weeks. For rechallenge experiments, DED mice resting for 7 weeks in the non-desiccated vivarium (after the initial desiccation) were placed back into the controlled-environment chamber for additional 7 days. Corneal epithelial disease was evaluated using fluorescein
(Sigma-Aldrich) staining and scored using the National Eye Institute grading system. ${ }^{31}$ In the in vivo blocking experiments, DED mice were treated with $100 \mu \mathrm{g}$ of monoclonal anti-IL-23R Ab (Clone 258010, R\&D Systems) or control IgG (R\&D Systems) intraperitoneally at day $14 .^{12}$ 
Fig. 6 IL-23 is required for the in vivo generation of memory Th17 cells from its effector precursor. Wild-type CD45.1 $1^{+}$B6 mice were challenged with desiccating stress for 14 days to induce acute DED, and then removed from the stress and received adoptive transfer of $1 \times$ $10^{7}$ congenic CD45.2 ${ }^{+} \mathrm{CD} 44^{\circ} \mathrm{CD}^{\circ} \mathrm{L}^{-} \mathrm{CD} 4^{+}$effector DED T cells at D14. Immediately after T cell transfer, the recipients received the anti-IL-23R antibody or isotype lgG treatment. Seven days later, the transferred congenic CD45.2 $2^{+} \mathrm{T}$ cell were examined in the recipients. Data are representative of two independent experiments with five animals in each experiment. a The numbers of congenic CD4 ${ }^{+} \mathrm{T}_{\text {cells }}$ in draining lymph nodes (DLN) were calculated based on the quantified frequencies using flow cytometry analysis. Results are expressed in the bar graph as mean \pm SEM. AT adoptive transfer of congenic T cells. Frequencies of congenic total Th17 (b) and memory Th17 cells (c) in the recipients' DLN were analyzed. Memory Th17 (mTh17) versus effector Th17 (eTh17) were differentiated based on the expression of IL-7R and CD44. Data shown in the bar graphs represent the mean \pm SEM. ns not significant by one-way ANOVA followed by Bonferroni's multiple comparisons post hoc test $(\mathbf{a})$ or by Student's $t$ test $(\mathbf{b}, \mathbf{c}) .{ }^{*} p<0.05 ;{ }^{* *} p<0.001$. d-f The conjunctival tissues were examined for infiltration of transferred congenic $\mathrm{CD}^{+}(\mathbf{d})$, total Th17 (e), and memory Th17 cells (f) by flow cytometry analysis. FMO fluorescence minus one control. Data shown represent the mean \pm SEM of pooled independent experiments with five animals in each group in every experiment. ${ }^{* *} p<0.01 ;{ }^{* * *} p<0.001$ by Student's $t$ test.

Flow cytometry analysis and reagents

Single-cell suspensions were prepared from lymphoid tissues of mice using a 70- $\mu \mathrm{m}$ cell strainer (BD Biosciences). Conjunctival tissues were first digested in RPMI (Invitrogen) with $2 \mathrm{mg} / \mathrm{ml}$ DNase and $2 \mathrm{mg} / \mathrm{ml}$ Collagenase (Roche) at $37^{\circ} \mathrm{C}$. The following Abs were used for flow cytometry analysis: FITC-anti-CD4 (clone RM4-5), PerCP-Cy5.5-anti-CD44 (clone IM7), PE-anti-IL-7Ra (clone A7R34), APC-anti-IL-15Ra (clone 6B4C88), PE-anti-CD62L (clone MEL-14), Brilliant Violet 421-Annexin V (all above from BioLegend), PE-Cy7-anti-IL-17, and PE-anti-IL-17 (clone eBio17B7, eBioscience). For intracellular IL-17 staining, cells were stimulated with phorbol 12-myristate 13-acetate and ionomycin (SigmaAldrich) for $6 \mathrm{~h}$ in the presence of GolgiStop ${ }^{T M}$ (BD Biosciences). Stained cell were examined with an LSR II flow cytometer (BD Biosciences), and the results were analyzed using FlowJo V10 software (Tree Star).

Cell sorting and culture

Draining lymph nodes from DED mice were harvested and pooled, and $\mathrm{CD}^{+}{ }^{+}$cells were isolated via negative selection with a $\mathrm{CD} 4^{+}$ T cell isolation kit (Miltenyi Biotec Inc). The isolated cells were $>98 \% \mathrm{CD}^{+}$as confirmed by flow cytometry, and they were stained with FITC-anti-CD44 (clone IM7) and PE-anti-CD62L (clone MEL-14, BioLegend) antibodies, and sorted for $\mathrm{CD} 44^{10} \mathrm{CD}^{\mathrm{O}} 2 \mathrm{~L}^{-}$ effector subpopulation using a MoFlo ${ }^{\oplus}$ FACS sorter (Dako Cytomation). The sorted $\mathrm{CD} 44^{\mathrm{lo}} \mathrm{CD} 2 \mathrm{~L}^{-} \mathrm{CD} 4^{+}$cells were treated with IL-23 (10 ng/ml, eBioscience), IL-2 (50 IU/ml, Pepro Tech), IL23+IL-2, monoclonal CD25 blocking Ab (CD25 mAb, $10 \mu \mathrm{g} / \mathrm{ml}$, clone PC61.5, eBioscience), or IL-23+CD25 mAb for $48 \mathrm{~h}$. Memory Th17 cells, as well as expression levels of IL-7R, IL-15R, and Annexin $\mathrm{V}$, were then examined by flow cytometry.

\section{T cell adoptive transfer}

The $\mathrm{CD} 44^{\mathrm{l}} \mathrm{CD} 2 \mathrm{~L}^{-} \mathrm{CD} 4^{+}$effector $\mathrm{T}$ cells were sorted as described above. In all, $1 \times 10^{7}$ cells from CD45.2 $2^{+}$DED mice were subsequently injected intravenously into congenic CD45.1 $1^{+}$DED mice, which also received $100 \mu \mathrm{g}$ of monoclonal anti-IL-23R Ab (Clone 258010, R\&D Systems) or control IgG (R\&D Systems) intraperitoneally immediately after the cell transfer.

\section{Real-time PCR}

Draining lymph nodes and conjunctivae from mice were harvested, frozen in TRIzol Reagent (Invitrogen Corp), and stored at $-80^{\circ} \mathrm{C}$ until use. Total RNA was isolated with an RNeasy ${ }^{\oplus}$ Micro kit (Qiagen) according to the manufacturer's recommendations and reverse transcribed using a QuantiTect Reverse Transcription Kit (Qiagen). Real-time PCR was performed using TaqMan Universal PCR Master Mix and predesigned primers for IL-23 (Mm00518984_m1), IL-2 (Mm00434256_m1), and GAPDH (Mm99999915_g1) (Applied Biosystems) in a Mastercycler ep realplex (Eppendorf). The GAPDH gene was used as an endogenous control for each reaction. The results of quantitative PCR were analyzed by the comparative CT method in which the target change $=2^{-\Delta \Delta C T}$. The results were normalized by the $\mathrm{CT}$ value of GAPDH, and the mean $\mathrm{CT}$ of relative mRNA level in the normal group was used as the calibrator.

ELISA

For protein extraction, draining lymph nodes and conjunctivae were harvested and stored in cold sterile PBS containing protease inhibitors (Sigma-Aldrich) at $-80^{\circ} \mathrm{C}$ until used. The samples were homogenized on ice and centrifuged. The supernatant was assayed for total protein levels using Pierce ${ }^{\mathrm{TM}}$ BCA Protein Assay Kit (Thermo Fisher Scientific), and for the levels of IL-23, IL-2, and IL-17 using commercial ELISA kits (eBioscience). The results of the cytokine levels were calibrated to the equal amount of total tissue proteins.

\section{Statistical analysis}

For comparison of multiple groups with normally distributed data, the statistical significance of endpoints was evaluated by one-way ANOVA followed by Bonferroni's multiple comparisons post hoc test. For comparison of two groups with normally distributed data, the unpaired two-tailed Student's $t$ test was used. All statistical analyses were performed with Prism software (version 5; GraphPad Software), and differences were considered significant at $p<0.05$.

Supplementary Material is linked to the online version of the paper at http://www.nature.com/mi.

\section{ACKNOWLEDGEMENTS}

We thank Dr Karin Roesch (Ayoxxa Biosystems Inc) for helping with multiplex protein assay with LUNARIS ${ }^{\mathrm{TM}}$ Kits. This work was supported by NIH grant EY20889 (R.D.) and NIH core grant P30EY003790.

\section{AUTHOR CONTRIBUTIONS}

Y.C. and C.S. designed and performed experiments and analyzed data. Y.C. also wrote the paper. N.W.F. performed critical in vivo experiments. T.N. contributed to in vivo treatment experiments. A.A. provided critical advice to the project and edited the paper. S.K.C. designed experiments and provided critical advice to the project. R.D. designed experiments and wrote the paper.

\section{ADDITIONAL INFORMATION}

The online version of this article (https://doi.org/10.1038/s41385-020-0289-3) contains supplementary material, which is available to authorized users.

Competing interests: The authors declare no competing interests.

Publisher's note Springer Nature remains neutral with regard to jurisdictional claims in published maps and institutional affiliations.

\section{REFERENCES}

1. Nussenblatt, R. B. The natural history of uveitis. Int. Ophthalmol. 14, 303-308 (1990). 
The functions of IL-23 and IL-2 on driving autoimmune effector T-helper... $Y$ Chen et al.

2. Cornelissen, F. et al. IL-23 dependent and independent stages of experimental arthritis: no clinical effect of therapeutic IL-23p19 inhibition in collagen-induced arthritis. PLoS One 8, e57553 (2013).

3. Williams, M. A. \& Bevan, M. J. Effector and memory CTL differentiation. Annu. Rev. Immunol. 25, 171-192 (2007).

4. Zielinski, C. E. et al. Dissecting the human immunologic memory for pathogens. Immunol. Rev. 240, 40-51 (2011).

5. Hernandez-Santos, N. et al. Th17 cells confer long-term adaptive immunity to oral mucosal Candida albicans infections. Mucosal Immunol. 6, 900-910 (2013).

6. Kryczek, l. et al. Human Th17 cells are long-lived effector memory cells. Sci. Transl. Med. 3, 104ra100 (2011).

7. Muranski, P. et al. Th17 cells are long lived and retain a stem cell-like molecular signature. Immunity 35, 972-985 (2011).

8. Chen, Y., Chauhan, S. K., Lee, H. S., Saban, D. R. \& Dana, R. Chronic dry eye disease is principally mediated by effector memory Th17 cells. Mucosal Immunol. 7, 38-45 (2014).

9. Farrand, K. F., Fridman, M., Stillman, I. Ö. \& Schaumberg, D. A. Prevalence of diagnosed dry eye disease in the United States among adults aged 18 years and older. Am. J. Ophthalmol. 182, 90-98 (2017).

10. Chen, Y., Chauhan, S. K., Tan, X. \& Dana, R. Interleukin-7 and -15 maintain pathogenic memory Th17 cells in autoimmunity. J. Autoimmun. 77, 96-103 (2017).

11. Haines, C. J. et al. Autoimmune memory T helper 17 cell function and expansion are dependent on interleukin-23. Cell Rep. 3, 1378-1388 (2013).

12. Chen, Y. et al. IFN- $\gamma$-expressing Th17 cells are required for development of severe ocular surface autoimmunity. J. Immunol. 199, 1163-1169 (2017).

13. Pepper, M. \& Jenkins, M. K. Origins of CD4(+) effector and central memory T cells. Nat. Immunol. 12, 467-471 (2011).

14. Harrington, L. E., Janowski, K. M., Oliver, J. R., Zajac, A. J. \& Weaver, C. T. Memory CD4 T cells emerge from effector T-cell progenitors. Nature 452, 356-360 (2008).

15. Lohning, $M$. et al. Long-lived virus-reactive memory $T$ cells generated from purified cytokine-secreting T helper type 1 and type 2 effectors. J. Exp. Med. 205, 53-61 (2008).

16. Castro, I., Dee, M. J. \& Malek, T. R. Transient enhanced IL-2R signaling early during priming rapidly amplifies development of functional CD8+ T effector-memory cells. J. Immunol. 189, 4321-4330 (2012).
17. Cunin, $P$. et al. The tachykinins substance $P$ and hemokinin- 1 favor the generation of human memory Th17 cells by inducing IL-1 $\beta$, IL-23, and TNF-like 1A expression by monocytes. J. Immunol. 186, 4175-4182 (2011).

18. Ghoreschi, K. et al. Generation of pathogenic $T(H) 17$ cells in the absence of TGF- $\beta$ signalling. Nature 467, 967-971 (2010).

19. McGeachy, M. J. et al. TGF-beta and IL- 6 drive the production of IL-17 and IL-10 by $\mathrm{T}$ cells and restrain $\mathrm{T}(\mathrm{H})-17$ cell-mediated pathology. Nat. Immunol. 8, 1390-1397 (2007).

20. McGeachy, M. J. et al. The interleukin 23 receptor is essential for the terminal differentiation of interleukin 17-producing effector $\mathrm{T}$ helper cells in vivo. Nat. Immunol. 10, 314-324 (2009).

21. Stockinger, B. Good for goose, but not for gander: IL-2 interferes with Th17 differentiation. Immunity 26, 278-279 (2007).

22. Liao, W. et al. Priming for $T$ helper type 2 differentiation by interleukin 2-mediated induction of interleukin 4 receptor alpha-chain expression. Nat. Immunol. 9, 1288-1296 (2008).

23. Liao, W., Lin, J. X., Wang, L., Li, P. \& Leonard, W. J. Modulation of cytokine receptors by IL-2 broadly regulates differentiation into helper T cell lineages. Nat. Immunol. 12, 551-559 (2011).

24. Laurence, A. et al. Interleukin-2 signaling via STAT5 constrains T helper 17 cell generation. Immunity 26, 371-381 (2007).

25. Amadi-Obi, A. et al. TH17 cells contribute to uveitis and scleritis and are expanded by IL-2 and inhibited by IL-27/STAT1. Nat. Med. 13, 711-718 (2007).

26. Elson, C. O. et al. Monoclonal anti-interleukin 23 reverses active colitis in a T cellmediated model in mice. Gastroenterology 132, 2359-2370 (2007).

27. Waldmann, T. A. The multi-subunit interleukin-2 receptor. Annu. Rev. Biochem. 58, 875-911 (1989).

28. Dooms, H., Wolslegel, K., Lin, P. \& Abbas, A. K. Interleukin-2 enhances CD4+ T cell memory by promoting the generation of IL-7R alpha-expressing cells. J. Exp. Med. 204, 547-557 (2007).

29. McKinstry, K. K. et al. Effector CD4 T-cell transition to memory requires late cognate interactions that induce autocrine IL-2. Nat. Commun. 5, 5377 (2014).

30. Kalia, V. et al. Prolonged interleukin-2Ralpha expression on virus-specific CD8+ T cells favors terminal-effector differentiation in vivo. Immunity 32, 91-103 (2010).

31. Lemp, M. A. Report of the National Eye Institute/Industry workshop on clinical trials in dry eyes. CLAO J. 21, 221-232 (1995). 